\title{
El termalismo y culto a las aguas en la Prensa médica Española
}

\author{
Francisco Maraver Eyzaguirre *
}

\section{INTRODUCCIÓN}

Desde el punto de vista del profesional de la medicina especializado en Hidrología, el tema del Termalismo y culto a las aguas, es muy sugestivo; tanto es así, que aparece reflejado en la mayoria de los Tratados - Manuales de la especialidad, al ocuparse de los antecedentes históricos ${ }^{1}$.

En nuestro pais este interés se hace aún más evidente, en la medida en que encontramos restos de auténticas ciudades termales en el mismo centro urbano de poblaciones como: Caldas de Malavella (Girona), Caldas de Montbuy (Barcelona); o establecimientos balnearios que utilizan las mismas instalaciones que en épocas remotas: Alange (Badajoz), Caldas de Lugo (Lugo), Montemayor (Cáceres); y otros como: Archena (Murcia), Retortillo (Salamanca)... que muestran con orgullo, en lugares preferentes vestigios de su pasado.

En relación a nuestra labor asistencial desde hace doce años, en el equipo de Dirección del Balneario de Baños de Montemayor, al realizar nuestra tesis doctoral sobre el citado centro termal, y ocuparnos del encuadre histórico, descubrimos, que eran numerosos los expertos en ar-

* Facultad de Medicina, Universidad Complutense de Madrid.

1 Acerca de la historia antigua de la Hidrología Médica vide PAzzinı, A. (1950), «Storia delle cure idrologiche e climatologiche», en MESSINI, M. (dir.) Trattato di Idroclimatologia Clinica. Bolonia, Cappelli Ed., págs. 13-185, 56 figs.; Acclaluolı, L. de M. C. (1952), “L'Évolution de la Crénotechnie», en Le Potugal Hydromineral. Lisboa, Dirección General de Minas y de Servicios Geológicos, págs. 1-112, 90 figs. y Armiluo Valenzuela, M. (1968), "Concepto y evolución de la Hidrología médica", en Compendio de Hidrología médica. Barcelona, Ed. Científico-Médica, págs. 1-12, 6 figs. 
queología que se habian interesado por dicho establecimiento ${ }^{2}$. Así mismo, fueron de inestimable ayuda las Memorias Oficiales manuscritas e impresas de sus Médicos Directores que desempeñaron el cargo desde $1818^{3}$, llamándonos poderosamente la atención, que los primeros ignorasen la aportación de éstos y viceversa.

Lo anterior, creemos, es consecuencia directa del estado lamentable en que se encontraban las instalaciones termales españolas a finales del siglo xvIII. Esto se deduce de las obras de carácter general de autores como: Limón Montero, Gómez de Bedoya, Garcia Ayuda, y de estudios posteriores de la época ${ }^{4}$.

${ }^{2}$ Cabe destacar, aparte del Corpus Inscriptionum latinarum II, Viu, J. de, Extremadura. Sus inscripciones y monumentos. Madrid 1852; FITA r COLOME, F., "Excursiones epigráficas", Boletín de la Real Academia de la Historia, vol. XXV. Madrid 1894, págs. 146-151; RoLDÁN Hervás, J. M., "Las lápidas votivas de Baños de Montemayor», Zephyrvs, vol. XVI. Salamanca 1965, págs. 5-57 y HABA Quirós, S., Catálogo epigráfico latino del partido judicial de Plasencia. Memoria de Licenciatura inédita, Universidad de Extremadura. Cáceres 1986. Estos trabajos se suceden de manera muy similar en toda la Comunidad, Ortiz Romero, P., Introducción a una historia de la Arqueologia en Extremadura. Cáceres, Universidad de Extremadura, 1986, 127 págs.

${ }^{3}$ Entre las impresas cabe destacar las de Rodriguez Solano, C., Manual del enfermo bañista en el establecimiento de aguas medicinal Baños en la provincia de Cáceres. Plasencia, Imprenta de don Manuel Ramos, 1838, 30 págs.; Martínez Serrano, F., Investigaciones hidrológicas en particular sobre el manantial termal del pueblo de Baños de MonteMayor y Béjar divididas en varias memorias. Cuarta memoria perteneciente al año de 1842. Plasencia, Imprenta de don Manuel Ramos, 1843, 111 págs.; ibidem, Sesta Memoria perteneciente al año de 1844. Cáceres, Imprenta de don Lucas de Burgos, 1845, 80 págs.; ibidem, Séptima Memoria perteneciente al año de 1845. Cáceres, Imprenta de don Lucas de Burgos, 1845, 116 págs.; Rodriguez Solano, C., Virtudes medicinales y análisis del agua minero-medicinal de Baños. Cáceres, Imprenta de Concha y Compañía, 1850, 22 págs.; JIMÉNEZ Y SÁNCHEz, P., Memoria acerca de las aguas minero-medicinales del pueblo de Baños de Montemayor y Béjar. Madrid, Imprenta de Manuel de Rojas, 1862, 17 págs.; Cordova Y Yécona, T., Noticias sobre el establecimiento de Baños minero-medicinales sulfurosos termales de Montemayor. Madrid, Imprenta del Colegio de sordo-mudos y de ciegos, 1864, 48 págs.; Crespo y EscoriazA, B., Breve reseña de las aguas sulfurados-sódicas termales de Montemayor o Baños. Béjar, Imp. de F. Aguilar y Álvarez, 1889, 24 págs.; ibidem, Aguas sulfuradosódicas termales de Montemayor ó Baños. Madrid, Establecimiento tipográfico de J. Palacios, 1899, 56 págs. y GurRucharri y EchaurI, J. E., Memoria quinquenal de las aguas minerales de Baños de Montemayor, (inédita, mecanografiada), Archivo central de la Facultad de Medicina de la U.C.M., 1916, 143 págs.

${ }^{4}$ Carretero y Muriel, M., Estado de la Hidrologia Médica Española en los siglos xvil y xvim. Madrid, Discurso inaugural de 1894 de la Real Academia de Medicina, Establecimiento tipográfico de Enrique Teodoro, 1894, 33 págs.; Miralles, J. P., «Contribución al estudio de la Hidrología médica española», Trabajos de la Cátedra Historia critica de la Medicina, t. III. Madrid 1934, 203-211; MÁlAGA GuerReRo, S., "La Hidrología Española del siglo XVIII", Cuadernos de Historia de la Medicina Española, núm. VIII. Salamanca 1969, 169-218 y Gran.JeL, L. S., "Limón Montero y la literatura hidrológica española del siglo XVII", Cuadernos de Historia de la Medicina Española, núm. XII. Salamanca 1973, 265-277. 
«Generalmente, los medios balneoterápicos consistían en albercas, balsas, pilones y estanques, casi siempre al aire libre, pues estaban cubiertas las piscinas en corto número de establecimientos, como sucedía en Arnedillo, Montemayor, Alhama de Granada y Aragón y Ledesma. En tales Baños se sumergían sin distinción toda clase de enfermos, estando mezclados los reumáticos y paralíticos con los afectados de dolencias contagiosas y repugnantes, sin que hubiera encargado que corrigiera tan grave desorden» ${ }^{5}$.

Esta situación cambia paulatinamente a lo largo del siglo $x i x$, en que se produce el desarrollo de la Hidrología Médica nacional, tanto en el terreno material, como se refleja ya en la obra de $\mathrm{MADOZ}^{6}$; con sucesivas reformas en los establecimientos Balnearios, (momento en el que aparecen numerosos restos arqueológicos y numismáticos), como en el terreno científico, con la creación en 1816, por Fernando VII, del Cuerpo de Médicos de Baños ${ }^{7}$.

«Entre los muchos y preciosos dones con que la Providencia favoreció a la España, debe considerarse por uno de los principales la abundancia de aguas minerales que distribuyó en varios puntos de su vasta extensión, ..., He venido en resolver que en cada uno de los Baños más acreditados del reino se establezca un profesor de suficientes conocimientos de las virtudes de sus aguas, y de la parte médica necesaria para saber determinar su aplicación y uso ... y se encargará a quién corresponda, que desde el día en que llegue a cada uno de los Baños el Profesor destinado, no se permita a ningún enfermo el uso de ellos sino con su permiso, $y$ en los términos que prescriba. Tendréislo entendido y dispondréis lo necesario al cumplimiento. Rubricado de la Real mano de su S.M. En Palacio a 29 de junio de 1816. A. D. Pedro Ceba$\|$ IOS ${ }^{8}$.

\footnotetext{
${ }^{5}$ Carretero y Muriel, M., Op. cit., pág. 31.

${ }^{6}$ Pineda Dorado, M. C., Balnearios en la España del Siglo xix según la obra de don Pascual Madoz. Memoria de Licenciatura, inédita, Universidad de Sevilla, 1986.

7 Sobre la creación del Cuerpo de Médicos de Baños vide Manzaneque, M., 1916, Primer centenario de la creación del cuerpo de Médico-Directores de Baños. Madrid, Imprenta de R. Rojas; Rodriguez Pinilla, H., "Sobre Historia de la Hidrología española», Anales de la Sociedad Española de Hidrología Médica, vol. XXX. Madrid 1919, págs. 27-33; DE Dios MATEO, R., La creación del Cuerpo de Médicos de Baños. Memoria de Licenciatura, Universidad de Salamanca. Salamanca 1969 y Garcia-Talavera Fernández, J. R., "Historia del Cuerpo de Médicos de Baños. Siglo XIX", Cuadernos de Historia de la Medicina Española, vol. X. Salamanca 1971, págs. 213-281.

${ }^{8}$ Decretos del Rey don Fernando VII, citado por Garcla-Talavera Fernández, J. R., Op. cit., págs. 215-216.
} 
De los sucesivos reglamentos que desde 1817 han regulado el anterior Real Decreto cabe destacar la obligación de los citados facultativos de realizar una Memoria anual en la que se reflejaban entre otras cosas el estado de las instalaciones, reformas llevadas a cabo y mejoras necesarias. Con esta actuación los Médicos Directores actuaron, al realizar este cometido, como notarios puntuales de todos los acontecimientos y hallazgos que se producian en los centros que dirigian.

De ahí la importancia, a nuestro juicio, de la utilización por los profesionales de la historia de estas fuentes que pueden aportar datos interesantes a su labor, como en el caso antes referido de Baños de Montemayor.

Desde el punto de vista científico otro hecho notable lo constituyó la creación de la Sociedad Española de Hidrología Médica ${ }^{9}$.

De todo lo anterior puede deducirse la importancia que tiene para el estudio histórico de cualquier agua mineromedicinal, la monumental obra de Martínez Regera, Bibliografia Hidrológico-Médica Española ${ }^{10}$, de finales del siglo pasado. Los fondos en ella reflejados, pueden consultarse en las instituciones siguientes: Archivo Histórico Nacional, Biblioteca Nacional, Biblioteca de la Facultad de Medicina de la U.C.M., Biblioteca de la Real Academia de Medicina, Biblioteca de la Real Academia de Farmacia, Biblioteca del Palacio Real de Madrid, Biblioteca del Ateneo de Madrid, Biblioteca del llustre Colegio de Médicos de la Autonomía de Madrid, así como los fondos del antiguo Ministerio de la Gobernación.

Por otra parte, no es necesario resaltar la importancia que tienen las publicaciones periódicas, como fuentes historiográficas en la elaboración de la Historia; es por lo que nos pareció oportuno hacer una revisión de

\footnotetext{
9 Acerca de la creación de la Sociedad Española de Hidrología Médica, vide «Sociedad Española de Hidrologia Médica. Sesión inaugural del 26 de febrero de 1876", Anales de la Sociedad Española de Hidrologia Médica, vol. I. Madrid 1877, págs. 25-35; SAN José RodriGUEZ, „. C., «Sociedad Española de Hidrología Médica. Datos y fechas», Boletín de la Sociedad Española de Hidrología Médica, vol. I. Madrid 1986, págs. 5-10 y Armijo Valenzuela, M., "Antecedentes históricos y evolución de la Especialidad», en ARmlso, M. et alii, Hidrología. Fuenlabrada (Madrid), Organización Médica Colegial 1990, págs. 1-25.

10 Martínez Regera, L., Bibliografía Hidrológico-Médica Española (Sección Impresos). Madrid, Imp. y Fund. de M. Tello, 1892, 954 págs.; ibidem, Bibliografia Hidrológico-Médica Española, segunda parte (Manuscritos y Biografias). Madrid, Establecimiento Tipográfico «Sucesores de Rivadeneyra», 1896, t. 1., 636 págs. e ibidem, Bibliografia Hidrológico-Médica Española, segunda parte (Manuscritos y Biografías). Madrid, Establecimiento Tipográfico "Sucesores de Rivadeneyra», 1897, t. 2., 883 págs.
} 
la Prensa Médica Española ", en relación con el Termalismo y Culto a las Aguas, objeto del presente trabajo, y más concretamente de las disciplinas: Hidrología Médica e Historia de la Medicina.

\section{Material y método}

El material utilizado está constituido, fundamentalmente, por las colecciones de publicaciones periódicas de Hidrologia Médica e Historia de la Medicina.

Las de Hidrologia Médica ${ }^{12}$ son:

Anales de la Sociedad Española de Hidrología Médica (1877-1932)

Órgano de expresión de la Sociedad Española de Hidrología Médica, desde el año siguiente a su constitución hasta la disolución del Cuerpo de Médicos de Baños, por Decreto en el período republicano.

Boletín Español de Hidrología Médica y Climatología (1950-1952)

Órgano de expresión de la Sociedad Española de Hidrología Médica, en su segunda época.

"1 Sobre la Prensa Médica Española, vide Méndez Álvaro, F., Breves apuntes para la historia del periodismo médico y farmacéutico en España. Madrid, Imp. E. Teodoro, 1883; LaRRa y Cerezo, A., Historia resumida del periodismo médico en España. Madrid 1905; CoRTEzo Collantes, A., «Historia del periodismo médico en España. El Siglo Médico y la evolución científica y profesional», El Siglo Médico, vol. LXVI, núm. 3.410. Madrid 1919, págs. 313319; RierA, J., «Francisco Méndez Álvaro. Historia del Periodismo Médico y Farmacéutico en España (introducción, edición e indices de J. RIERA)", Acta Histórico-Médica Vallisoletana, vol. VIII. Valladolid 1978, págs. 13-19 y López PIÑERo, J. M. y TerRAda, M. L., «Las etapas históricas del periodismo médico en España. Estudio bibliométrico", en AlbarRAcin, A.; LópEz Piñero, J. M. y Granjel, L. S. (edit.) 1980: Medicina e Historia. Madrid, Ed. de la Universidad Compiutense, págs. 163-191.

${ }^{12}$ Garcia de Léaniz Garzon, J., «Sociedad Española de Hidrología Médica (Anales y Boletines)", Boletín de la Sociedad Española de Hidrologia Médica, vol. II, núm. 1. Madrid 1987, págs. 5-7 y Maraver Eyzaguirre, F. y Corvillo Martín, I., «La Publicación científica especializada" en Armiso, M. et alii, 1990, Hidrología. Madrid, Organización Médica Colegial, págs. 45-55. 
Anales Hispanoamericanos de Hidrología Médica y Climatología (19541959)

Órgano de expresión del desaparecido Instituto Limón Montero de Hidrología y Climatología Médica, del C.S.I.C.

Boletín de la Sociedad Española de Hidrología Médica (1962-1964)

Órgano de expresión de la Sociedad Española de Hidrología Médica, en su tercera época.

Anales de la Real Academia de Farmacia (1968...)

Serie monográfica dedicada a las aguas mineromedicinales, órgano de expresión de la Comisión para el estudio de las aguas medicinales de la Real Academia de Farmacia, patrocinado por el Instituto de España ${ }^{13}$.

Boletín de la Sociedad Española de Hidrologia Médica (1986...)

Órgano de expresión de la Sociedad Española de Hidrologia Médica, en su cuarta época.

La totalidad de estas publicaciones se han editado en Madrid.

Las de Historia de la Medicina son:

Trabajos de la Cátedra de Historia Critica de la Medicina (1932-1935)

Órgano de expresión de la Cátedra de Historia de la Medicina de la Facultad de Medicina de la antigua Universidad Central. Se editan en Madrid.

${ }^{13}$ Las publicadas hasta la fecha son: Caldas de Tuy, 1968; Caldas de Cuntis, 1974; Montemayor, 1975; Corconte, 1976; Ledesma, 1977; Solán de Cabras, 1978; Lanjarón, 1980; Carabaña, 1981; Alhama de Aragón, 1983; Caldas de Montbuy, 1984; Fuente Amarga de Chiclana, 1985; Archena, 1986; Fortuna, 1987; Arnedillo, 1988; Caldas de Bohi, 1989 y Alange, 1990. 
Asclepio, Revista de Historia de la Medicina y de la Ciencia (1949...)

Órgano de expresión del Centro de Estudios Históricos del C.S.I.C. Esta revista empezó a publicarse, en 1949, con la denominación de Archivos Iberoamericanos de Historia de la Medicina, como órgano de expresión del Instituto «Arnau de Vilanova» de Historia de la Medicina, hasta 1954 en que pasó a llamarse Archivos Iberoamericanos de Historia de la Medicina y Antropologia Médica, para tomar en 1964 su actual denominación. Se editan en Madrid ${ }^{14}$.

Cuadernos de Historia de la Medicina Española (1962-1975)

Órgano de expresión de la Cátedra de Historia de la Medicina de la Facultad de Medicina de la Universidad de Salamanca. Se editan en Salamanca.

Medicina e Historia (1964-1971; 1971-1978; 1984...).

Órgano de expresión del Centro de Documentación de Historia de la Medicina de los laboratorios Biohorm. Se han publicado en tres épocas diferentes y se editan en Barcelona.

Dynamis (1981...)

Órgano de expresión de la Unidad Docente de Historia de la Medicina de la Facultad de Medicina de la Universidad de Granada. Se editan en Granada.

Independientemente de las publicaciones de Hidrologia Médica e Historia de la Medicina, hemos utilizado la revista:

Medicina Española (1937-1985)

Revista Nacional de Medicina, Cirugia y Especialidades. Se editaba en Valencia. Sirvió de órgano de expresión a numerosos trabajos de la Cá-

\footnotetext{
14 Albarracin Teulón, A., "Conmemoración», Asclepio, vol. XXV. Madrid, págs. 3-6.
} 
tedra de Historia de la Medicina de la Facultad de Medicina de Valencia e Instituto de Historia de la Medicina de la Institución «Alfonso el Magnánimo», directamente relacionados con el tema que nos ocupa ${ }^{15}$.

El método utilizado consistió en la revisión exhaustiva y selección de los artículos publicados, directamente relacionados con el Termalismo y Culto a las Aguas, para posteriormente, con los resultados de nuestro trabajo, desarrollar la discusión y alcanzar las conclusiones pertinentes.

\section{RESULTADOS}

Fruto de esta revisión hemos localizado los siguientes trabajos que enumeramos por orden cronológico:

1. Bassadone, E., «Magia y Medicina», Trabajos de la Cátedra de Historia Critica de la Medicina, t. III. Madrid 1934, págs. 151-182.

2. García Álvarez, M. R., «La medicina en la Galicia prerromana», Asclepio, t. III, fasc. 1. Madrid 1951, págs. 275-293.

3. Castillo de lucas, A., «El Doctor Leite de Vasconcellos. Historiador de la Medicina Arcaica Portuguesa», Asclepio, t. X, fasc. 3. Madrid 1958, págs. 163-167.

\footnotetext{
is Zaragoza Rubira, J. R., "Aspectos Médicos de la España Primitiva en la "Historia Natural" de Plinio", Medicina Española, t. XLVIII, núm. 284. Valencia 1962, págs. 415-423; ibidem, "Aspectos Médicos de la España Primitiva en la "Geografía" de Estrabón", Asclepio, t. XVI. Madrid 1964, págs. 205-213; ibidem 1965, La Medicina en la España Antigua, Tesis Doctoral, Universidad de Valencia; ibidem, "La Medicina en la España Antigua", Cuadernos de Historia de la Medicina Española, año IV. Salamanca 1965, págs. 131-189; ibidem, "La medicina en Tartessos», Medicina Española, t. LV, núm. 323. Valencia 1966, págs. 138-146; ibidem, "La Medicina en la España Celta", Medicina Española, t. LVI, núm. 328. Valencia 1966, págs. 48-64; ibidem, «La Medicina de los Celtíberos", Medicina Española, t. LVI, núm. 330. Valencia 1966, págs. 185-194; ibidem, "La Medicina de la España protohistórica (Las civilizaciones autóctonas)", Cuadernos Valencianos de Historia de la Medicina y de la Ciencia, t. V. Valencia 1967, 68 págs.; ibidem, "La medicina de la España Púnica», Medicina Española, t. LVIII, núm. 343. Valencia 1967, págs. 254-278; ibidem, "La medicina de la España Griega", Medicina Española, t. LVIII, núm. 344. Valencia 1967, págs. 307-312; ibidem, "La medicina de la conquista Romana", Medicina Española, t. LVIII, núm. 345. Valencia 1967, págs. 374-395 e ibidem, «La Medicina de la España Prehistórica», Cuadernos de Historia de la Medicina Española, año XI. Salamanca 1972, págs. 345-357.
} 
4. Castillo de Lucas, A., «La Patera de Otañes», Anales Hispanoamericanos de Hidrología Médica y Climatología, t. III. Madrid 1959, págs. 413-424.

5. Zaragoza Rubira, J. R., «Aspectos Médicos de la España primitiva en la "Historia Natural" de Plinio", Medicina Española, t. XLVIII, núm. 284. Valencia 1962, págs. 415-423.

6. Zaragoza Rubira, J. R., "Aspectos Médicos de la España Primitiva en la "Geografia de Estrabón", Asclepio, t. XVI. Madrid 1964, págs. 205-213.

7. Zaragoza Rubira, J. R., "La Medicina en la España Antigua», Cuadernos de la Historia de la Medicina, año IV. Salamanca 1965, págs. 131-189.

8. Zaragoza Rubira, J. R., "La medicina de la España Celta", Medicina Española, t. LVI, núm. 328. Valencia 1966, págs. 48-64.

9. Zaragoza Rubira, J. R., «La medicina de la conquista Romana», Medicina Española, t. LVIII, núm. 345. Valencia 1967, págs. 374395.

10. Zaragoza Rubira, J. R., «La Medicina de la España Prehistórica», Cuadernos de Historia de la Medicina Española, año XI. Salamanca 1972, págs. 345-357.

11. LOPEZ DE AZCONA, J. M., "Consideraciones sobre el manantial minero-medicinal de Caldas de Montemayor", Anales de la Real Academia de Farmacia, núm. III. Madrid 1975, págs. 101-116.

12. LóPEZ DE AZCONA, J. M., «Consideraciones sobre el manantial minero-medicinal de Caldas de Montbui», Anales de la Real Academia de Farmacia, núm. X. Madrid 1984, págs. 5-22.

13. López de AzconA, J. M., "Consideraciones sobre el Baño de Archena", Anales de la Real Academia de Farmacia, núm. XII. Madrid 1986, págs. 5-21.

14. Ruiz Bremón, M., "Hidrología en el mundo ibérico; el Santuario del Cerro de los Santos", Boletín de la Sociedad Española de Hidrología Médica, vol. II, núm. 2. Madrid 1987, págs. 65-69.

15. Aguayo Martos, A., «Lápida de los Duunviros (Balneario de Archena)», Boletín de la Sociedad Española de Hidrología Médica, vol. II, núm. 3, págs. 115-118.

16. López de Azcona, J. M., «Comentarios sobre el Balneario de Fortuna", Anales de la Real Academia de Farmacia, núm. XIII. Madrid 1987, págs. 5-17. 
17. Gracia Guillén, D., «Lo físico y lo moral en la cura balnearia», Boletín de la Sociedad Española de Hidrología Médica, vol. III, núm. 1. Madrid 1988, págs. 5-10.

18. Haba, S.; Rodrígo, V. y Maraver, F., «Testimonios epigráficos del culto a las aguas en el área Caparense», Boletín de la Sociedad Española de Hidrología Médica, vol. Ill, núm. 2. Madrid 1988, págs. 63-66.

19. Gracia Guillen, D., «La Terapéutica por el agua», Boletín de la Sociedad Española de Hidrología Médica, vol. III, núm. 3. Madrid 1988, págs. 105-106.

20. Moltó, L., "Yacimientos termales de Hispania documentados por la Arqueología», Boletín de la Sociedad Española de Hidrología Médica, vol. V, núm. 3. Madrid 1990, págs. 103-107.

21. López de AzconA, J. M., «Consideraciones Generales sobre el balneario de Alange», Anales de la Real Academia de Farmacia, núm. XVI. Madrid 1990, págs. 5-26.

\section{DISCUSIÓN}

Hemos revisado 12 colecciones de publicaciones periódicas, seis de Hidrología Médica y seis de Historia de la Medicina, que abarcan un periodo de tiempo de 114 años (1877-1990) de la Prensa Médica española en estas disciplinas.

Fruto de estas pesquisas, es la selección de 21 artículos directamente relacionados con el tema «Termalismo y Culto a las aguas». Nueve de éstos, en cuatro publicaciones de Historia de la Medicina: Trabajos de la Cátedra de Historia crítica de la Medicina, Asclepio, Cuadernos de Historia de la Medicina Española y Medicina Española, y 12, en tres de Hidrología Médica: Anales Hispanoamericanos de Hidrología Médica y Climatología, Anales de la Real Academia de Farmacia y Boletín de la Sociedad Española de Hidrología Médica (4. Época). No hemos encontrado nada en: Medicina e Historia, Dynamis, Anales de la Sociedad Española de Hidrología Médica, Boletín de Hidrología Médica y Climatología y Boletín de la Sociedad Española de Hidrología Médica (3. Época).

Estos trabajos seleccionados ven la luz en un intervalo de 66 años (1934-1990), en el que distinguimos una primera época, anterior a 1972 , en que casi todos los artículos aparecen en publicaciones de Historia de 
la Medicina y, una segunda época, desde 1984, en que la totalidad figuran en las de Hidrología Médica.

Entre los autores, destacan por el número de obras: ZaRAGOZA RUBIRA, con seis; López de Azcona, con cinco; Castillo de lucas y Gracia Guillén, con dos, y el resto con una. Todos son profesionales de la medicina salvo López de Azcona, Ruiz Bremón, Haba y Rodrigo.

En primer lugar, llama la atención la labor de ZaRAGOza RuBiRa, que siendo secretario del instituto «Alfonso el Magnánimo» y ayudante de la Cátedra de Historia de la Medicina de la Universidad de Valencia, aborda todos los períodos de la Medicina de la España Antigua. Pone de manifiesto la asociación que tenía para los Iberos la práctica médica con aspectos mágico creenciales, así como la proximidad de sus Santuarios a manantiales, donde se han recogido numerosos exvotos ${ }^{16}$. De los pueblos de origen Celta, resalta el culto que daban a las fuentes y lagos, al tiempo que sus Druidas conocian y apreciaban las virtudes curativas de las aguas minero-medicinales ${ }^{17}$. En otros trabajos destaca la asociación de Fuentes como origen de augurios o presagios, según creencias populares de la época ${ }^{18} \mathrm{o}$ el empleo de prácticas termoterápicas, como los baños de vapor, echando agua encima de piedras enrojecidas por el fuego ${ }^{19}, y$ como por último, ya en la medicina de la España Romana, los colonizadores yuxtapusieron las creencias y prácticas de la población indígena a las suyas propias, cen lo que se desarrolló la devoción a los dioses curativos y a deidades acuáticas, sobre todo, Ninfas de ríos y manantiales de aguas minero-medicinales ${ }^{20}$.

En segundo lugar, consideramos la aportación de LÓPEZ DE AZCONA, doctor ingeniero de Minas, presidente de la Comisión para las aguas minero-medicinales de la Real Academia de Farmacia, quien de forma superficial señala la existencia de restos arqueológicos en varios establecimientos ${ }^{21}$.

De los primeros años son los trabajos de CASTILLO DE LUCAS, profesor adjunto de Hidrología Médica, secretario científico de los Anales HispanoAmericanos de Hidrología y Climatología y Académico de número de la Real Academia de Farmacia. En uno se ocupa de Leite de Vasconcellos

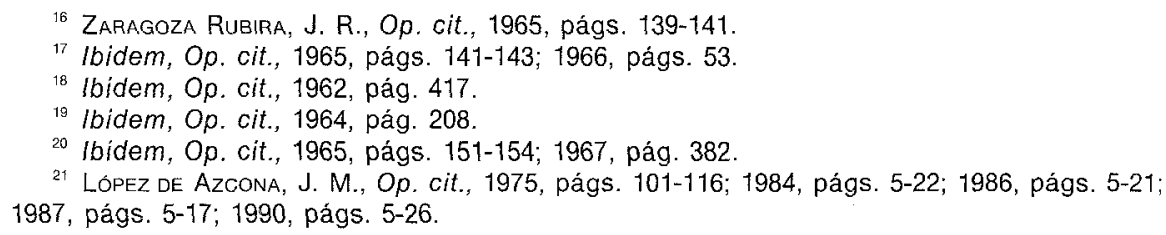

${ }^{21}$ López de Azcona, J. M., Op. cit., 1975, págs. 101-116; 1984, págs. 5-22; 1986, págs. 5-21; 1987, págs. 5-17; 1990, págs. 5-26. 
con motivo del primer centenario de su nacimiento, resaltando su aspecto de historiador de la medicina con la obra Medicina dos Lusitanos, donde destacaba las prácticas hidroterápicas y culto a las aguas del periodo prerromano $^{22}$, y otro, sobre la Patera de Otañes ${ }^{23}$.

Más recientes son los trabajos de Gracia GuILLEN ${ }^{24,25}$, catedrático de Historia de la Medicina de la Universidad Complutense, en los que considera la Medicina Antigua, como heredera de la tradición mágica y mítica, donde la enfermedad aguda, se asimilaba a castigo divino y a las aguas como fármacos por antonomasia de la misma y dignas de culto, de ahi el carácter purificador de la práctica crenoterápica.

Del resto de la primera época, citar los artículos de BASSADONE ${ }^{26}$ y García Álvarez ${ }^{27}$. El primero hace hincapié en el aspecto mágico de la medicina primitiva íntimamente asociada a la religión, y el segundo, profundiza sobre el estado de la medicina gallega antes de la llegada de las Legiones Romanas, la utilización de las aguas minerales y la multitud de restos epigráficos en relación a las divinidades (Bormánico, Edovio, Convertino...) en Vizela, Caldas de Reyes o Guitiriz, así como a las Ninfas en Caldas de Cuntis, Baños de Molgas y Orense.

Para finalizar, resaltar las publicaciones de: RUIZ BREMÓN ${ }^{28}$ que relaciona el Santuario Ibérico del Cerro de los Santos con las aguas mineromedicinales del Establecimiento Balneario de Baños de San José y con la «Sal de la Higuera» nombre farmacológico del sulfato de magnesio que se recoge en la zona que circunda al Cerro; AGuAYo ${ }^{29}$, médico-director durante muchos años del Balneario de Archena, que se ocupa del estudio de la lápida de los Duunviros que allí se conserva; HABA et alli ${ }^{30}$ que refiere los testimonios epigráficos del culto a las aguas en el área $\mathrm{Ca}$ parense, comarca vertebrada en unos $50 \mathrm{~km}$ por la ruta de la Plata, donde se encuentran de norte a sur los Balnearios de: Montemayor, El Salugral, Valdelazura y Baños de Cabezón, y Molto ${ }^{31}$ que estudia 46 yacimientos termales de la Península lbérica, conocidos y utilizados en la antigüedad, clasificándolos según la composición físico-química de sus aguas.

\footnotetext{
22 Castillo de lugas, A., Op. cit., 1958, págs. 363-365.

${ }^{23}$ Ibidem, Op. cit., 1959, págs. 413-424.

${ }^{24}$ Gracia Gullén, D., Op. cit, 1988, 1, págs. 5-10.

${ }^{25}$ Ibidem, Op. cit., 1988, 3, págs. 105-106.

${ }^{26}$ Bassadone, E., Op. cit., 1934, págs. 151-154.

${ }^{27}$ García Álvarez, M. R., Op. cit., 1951, págs. 289-292.

28 Ruiz Bremón, M., Op. cit., 1987, págs. 65-69.

${ }^{29}$ Aguayo Martos, A., Op. cit., págs. 115-118.

${ }^{30}$ Haba, S.; Rodrigo, V., y Maraver, F., 1988, págs. 63-66.

${ }^{31}$ Molto, L., Op. cit., 1990, págs. 103-107.
} 


\section{CONCLUSIONES}

De todo lo anterior podemos deducir las siguientes conclusiones:

1. ${ }^{a} \quad$ La importancia que tienen, a nuestro juicio, las Memorias impresas y manuscritas de los médicos directores que desempeñaron sus funciones en los respectivos balnearios, pues reflejan, en la mayoría de los casos puntualmente, la aparición de restos arqueológicos, epigráficos y numismáticos.

2. ${ }^{a} \quad$ El valor que representa la Prensa Médica española, concretamente en las disciplinas de Historia de la Medicina e Hidrología Médica, como fuente historiográfica, en relación a las aguas mineromedicinales y el culto a las mismas.

3. ${ }^{a} \quad$ En definitiva lo que hemos pretendido es familiarizar a los profesionales de la Historia y principalmente a los arqueólogos, con fuentes habitualmente poco consultadas, pero que consideramos de gran interés.

\section{RESUMEN}

Se pretende familiarizar a los profesionales de la Historia con fuentes poco utilizadas habitualmente pero, a nuestro juicio, interesantes.

En primer lugar, las Memorias impresas y manuscritas de los Médicos Directores del "Cuerpo de Baños", notarios puntuales de los acontecimientos y hallazgos que se producian en los centros que regentaban, y en segundo lugar, la Prensa Médica Española en relación con el culto a las aguas.

Para este último fin, se consideran 12 publicaciones periódicas de $\mathrm{Hi}$ drología Médica e Historia de la Medicina (años de publicación, instituciones que las han sustentado, etc.). Se estudian 21 artículos referidos al tema.

\section{ABSTRACT}

We intend to get familiar to historians with sources not too well employed by them, but in our opinion interesting. 
First of all, the printed and handwritten Memorials from medical managers of "Cuerpo de Baños", punctual lawyers of the events and discoveries produced in the Watering Bath Places managed by them. In second place, the Spanish Medical Press related with worship waters.

For this last purpose, we have considered twelve Hydrology Medical and History of Medicine periodical publications (years published, Institutions that have supported them, etc.). We have studied a total of 21 articles referred to this subject.

\section{BIBLIOGRAFIA}

Accialuol, L., Le Portugal Hydromineral. Lisboa, Dirección General de Minas y de Servicios Geológicos, 1952.

Aguayo Martos, A., "Lápida de los Duunviros (Balneario de Archena)", Boletín de la Sociedad Española de Hidrología Médica, vol. II, núm. 3. Madrid 1987, págs. 115-118.

Albarracín Teulón, A., "Conmemoración», Asclepio, vol. XXV. Madrid 1974, págs. 3-6.

Albarracin, A.; López Piñero, J. M. y Granjel, L. S. (edit.), Medicina e Historia. Madrid, Ed. de la Universidad Complutense, 1980.

armijo Valenzuela, M., Compendio de Hidrología Médica. Barcelona, Ed. Científico-Médica, 1968.

Armijo, M. et alii., Hidrología. Fuenlabrada (Madrid), Organización Médica Colegial, 1990.

Bassadone, E., «Magia y Medicina», Trabajos de la Cátedra de Historia Critica de la Medicina, t. III. Madrid 1934, págs. 151-182.

Carretero y Muriel, M., Estado de la Hidrología Médica Española en los siglos xvIf y xvim. Madrid, establecimiento tipográfico de Enrique Teodoro, 1894.

Castillo de lucas, A., «El doctor Leite de Vasconcellos. Historiador de la Medicina Arcaica Portuguesa", Asclepio, t. X, fasc. 3. Madrid 1958, págs. 153-167.

- :La Patera de Otañes», Anales Hispanoamericanos de Hidrologia Médica y Climatología t. IIl. Madrid 1959, págs. 413-424.

Códoba y Yécora, T., Noticias sobre el establecimiento de Baños minero-medicinales sulfurosos termales de Montemayor. Madrid, Imprenta del Colegio de sordo-mudos y de ciegos, 1864.

Cortezo Collantes, A., «Historia del Periodismo Médico en España. El Siglo Médico y la evolución científica y profesional», El Siglo Médico, vol. LXVI, núm. 3.410. Madrid 1919, págs. 313-319.

CREspo y EscoriazA, B., Breve reseña de las aguas sulfurado-sódicas termales de Montemayor o Baños. Béjar, Imp. de F. Aguilar y Álvarez, 1889.

-: Aguas sulfurado-sódicas termales de Montemayor o Baños. Madrid, establecimiento tipográfico de J. Palacios, 1899.

DE Dios Mateo, R. La creación del Cuerpo de Médicos de Baños. Memoria de Licenciatura inédita, Universidad de Salamanca, 1969.

Fita y Colomé, F.; "Excursiones epigráficas", Boletin de la Real Academia de la Historia, vol. XXV. Madrid 1894, págs. 146-151.

García Álvarez, M. R., "La medicina en la Galicia prerromana", Asclepio, t. III, fasc. 1. Madrid 1951, págs. 275-293. 
Garcia de Leaniz Garzón, J., "Sociedad Española de Hidrologia Médica (Anales y Boletines)", Boletin de la Sociedad Española de Hidrologia Médica, vol. II, núm. 1. Madrid 1987, págs. 5-7

Garcia Talavera, J. R., uHistoria del Cuerpo de Médicos de Baños. Siglo xix», Cuadernos de Historia de la Medicina Española, vol. X. Salamanca 1971, págs. 213-281.

Gracia Guillén, D., "Lo físico y lo moral en la cura balnearia», Boletin de la Sociedad Española de Hidrologia Médica, vol. III, núm. 1. Madrid 1988, págs. 5-10.

-: "La Terapéutica por el agua", Boletín de la Sociedad Española de Hidrología Médica, vol. III, núm. 3. Madrid 1988, págs. 105-106.

GRANJeL, L. S., "Limón Montero y la literatura hidrológica española del siglo XVII», Cuadernos de Historia de la Medicina Española, año XII. Salamanca 1973, págs. 265-277.

Gurrucharri y Echaurl, J. E., Memoria quinquenal de las aguas minerales de Baños de Montemayor (inédita, mecanografiada). Archivo central de la Facultad de Medicina de la U.C.M., 1916.

Haba QuiRós, S., Catálogo epigráfico latino del partido judicial de Plasencia. Memoria de Licenciatura inédita, Universidad de Extremadura, 1986.

Haba, S.; Rodrigo, V., y Maraver, F., "Testimonios epigráficos del culto a las aguas en el área Caparense", Boletín de la Sociedad Española de Hidrología Médica, vol. III, núm. 2. Madrid 1988, págs. 63-66.

Jiménez Y SÁnchez, P., Memoria acerca de las aguas minero-medicinales del pueblo de Baños de Montemayor y Béjar. Madrid, Imprenta de Manuel de Rojas, 1862.

LOPEZ DE AZCONA, J. M., "Consideraciones sobre el manantial minero-medicinal de Caldas de Montemayor", Anales de la Real Academia de Farmacia, núm. Ill. Madrid 1975, págs. $101-116$.

-: "Consideraciones sobre el manantial minero-medicinal de Caldas de Montbui", Anales de la Real Academia de Farmacia, núm. X. Madrid 1984, págs. 5-22.

-: "Consideraciones sobre el Baño de Archena", Anales de la Real Academia de Farmacia, núm. XII. Madrid 1986, págs. 5-21.

-: "Comentarios sobre el Balneario de Fortuna", Anales de la Real Academia de Farmacia, núm. XIII. Madrid 1987, págs. 5-17.

-: "Consideraciones Generales sobre el balneario de Alange", Anales de la Real Academia de Farmacia, núm. XVI. Madrid 1990, págs. 5-26.

Málaga Guerrero, S., "La Hidrología Española del siglo XVIIl», Cuadernos de Historia de la Medicina Española, núm. VIII. Salamanca 1969, págs. 169-218.

Manzaneque, M., Primer centenario de la creación del Cuerpo de Médicos-Directores de Baños. Madrid, Imprenta de R. Rojas, 1916.

Martinez Reguera, L., Bibliografia Hidrológico-Médica Española (sección impresos). Madrid, Imp. y Fund. de M. Tello, 1892.

-: Bibliografía Hidrológico-Médica Española, segunda parte (Manuscritos y Bibliografías). Madrid, establecimiento tipográfico "Sucesores de Rivadeneyra", t. 1. ${ }^{\circ}, 1896$.

-: Bibliografía Hidrológico-Médica Española, segunda parte (Manuscritos y Bibliografias). Madrid, estableciniiento tipográfico "Sucesores de Rivadeneyra", t. 2. ${ }^{\circ}, 1897$.

Martínez Serrano, F., Investigaciones hidrológicas en particular sobre el manantial termal del pueblo de Baños de Monte-Mayor y Béjar divididas en varias memorias. Cuarta memoria perteneciente al año de 1842. Plasencia, imprenta de don Manuel Ramos, 1843.

-: Sesta Memoria perteneciente al año de 1844. Cáceres, imprenta de don Lucas de Burgos, 1845.

—: Séptima Memoria perteneciente al año de 1845. Cáceres, imprenta de don Lucas de Burgos, 1845.

Méndez Álvaro, F., Breves apuntes para la historia del periodismo médico y farmacéutico en España. Madrid, Imp. E. Teodoro, 1883.

MessinI, M. (dir.), Trattato di Idroclimatologia Clinica. Bolonia, Cappelli Ed., 1950. 
Miralles, J. P., "Contribución al estudio de la Hidrología médica española», Trabajos de la Cátedra de Historia crítica de la Medicina, t. III. Madrid 1934, págs. 203-211.

MoLTO, L., "Yacimientos termales de Hispania documentados por la Arqueologia", Boletín de la Sociedad Española de Hidrología Médica, vol. V, núm. 3. Madrid 1990, págs. 103107.

Ortiz Romero, P., Introducción a una historia de la Arqueología en Extremadura. Cáceres, Universidad de Extremadura, 1986.

Pineda Dorado, M. C., Balnearios en la España del siglo xix según la obra de don Pascual Madoz. Memoria de Licenciatura inédita, Universidad de Sevilla, 1986.

RierA, J., "Francisco Méndez Álvaro. Historia del Periodismo Médico y Farmacéutico en España (introducción, edición e índices)", Acta Histórico-Médica Vallisoletana, vol. VIll. Valladolid 1978, págs. 13-19.

Rodriguez Pinilla, H., "Sobre la Historia de la Hidrología española", Anales de la Sociedad Española de Hidrología Médica, vol. XXX. Madrid 1919, págs. 27-33.

Rodriguez Solano, C.. Manual del enfermo bañista en el establecimiento de agua medicinal de Baños en la provincia de Cáceres. Plasencia, imprenta de don Manuel Ramos, 1838.

-: Virtudes medicinales y análisis del agua minero-medicinal de Baños. Cáceres, imprenta de Concha y compañia, 1850.

RoldÁN HeRVÁs, J. M., «Las lápidas votivas de Baños de Montemayor», Zephyrus, vol. XV1. Salamanca 1965, págs. 5-37.

Ruiz Bremón, M., "Hidrología en el mundo ibérico; el Santuario del Cerro de los Santos", Boletín de la Sociedad Española de Hidrologia Médica, vol. II, núm. 2. Madrid 1987, págs. $65-69$.

San José Rodriguez, J. C., "Sociedad Española de Hidrología Médica. Datos y fechas», Boletín de la Sociedad Española de Hidrología Médica, vol. I, núm. 1. Madrid 1986, págs. 5-10.

-: «Sociedad Española de Hidrologia Médica. Sesión inaugural del 26 de febrero de 1876", Anales de la Sociedad Española de Hidrologia Médica, vol. I. Madrid 1877, págs. 25-35.

Viu, J., Extremadura. Sus inscripciones y monumentos. Madrid 1852.

Zaragoza Rubira, J. R., "Aspectos médicos de la España primitiva en la "Historia Natural" de Plinio", Medicina Española, t. XLVIII, núm. 284. Valencia 1962, págs. 415-423.

-: "Aspectos Médicos de la España Primitiva en la "Geografía" de Estrabón", Asclepio, t. XVI. Madrid 1964, págs. 205-213.

-: "La Medicina en la España Antigua", Cuadernos de Historia de la Medicina Española, año IV. Salamanca 1965, págs. 131-189.

—: "La Medicina en Tartessos", Medicina Española, t. LV, núm. 323. Valencia 1966, págs. 138-146.

—: "La Medicina en la España Celta", Medicina Española, t. LVI, núm. 328. Valencia 1966, págs. 48-64.

-: "La Medicina de los Celtiberos», Medicina Española, t. LVI, núm. 330. Valencia 1966, págs. 185-194.

-: "La Medicina de la España protohistórica (las civilizaciones autóctonas)", Cuadernos Valencianos de Historia de la Medicina y de la Ciencia, t. V. Valencia 1967, 68 págs.

—: "La Medicina de la España Púnica", Medicina Española, t. LVIII, núm. 343. Valencia 1967, págs. 254-278.

-: «La Medicina de la España Griega», Medicina Española, t. LVIII, núm. 344. Valencia 1967, págs. 307-312.

-: «La Medicina de la conquista Romana», Medicina Española, t. LVIII, núm. 345. Valencia 1967, págs. 374-395.

-: «La Medicina de la España Prehistórica», Cuadernos de Historia de la Medicina Española, año XI. Salamanca 1972, págs. 345-357. 